\title{
II. THE RESEARCH ETHICS INVOLVING VULNERABLE GROUPS
}

\author{
Alejandra González-Duarte ${ }^{1 *}$, Elena Zambrano-GonzÁlez ${ }^{1}$, Heriberto Medina-Franco ${ }^{2}$, \\ Josefina Alberú-Gómez ${ }^{2}$, Marta Durand-Carbajal ${ }^{2}$, Carlos A. Hinojosa², Carlos A. Aguilar-Salinas ${ }^{2}$ \\ AND MARTHA Kaufer-HorWITZ², for the ${ }^{1}$ Ethics Committee and ${ }^{2}$ Research Committee of the \\ Instituto Nacional de Ciencias Médicas y Nutrición Salvador Zubirán, Mexico City, Mexico.
}

\begin{abstract}
Vulnerability in research occurs when the participant is incapable of protecting his or her interests and therefore, has an increased probability of being intentionally or unintentionally harmed. This manuscript aims to discuss the conditions that make a group vulnerable and the tools and requirements that can be used to reduce the ethical breaches when including them in research protocols. The vulnerability can be due either to an inability to understand and give informed consent or to unequal power relationships that hinder basic rights. Excluding subjects from research for the only reason of belonging to a vulnerable group is unethical and will bias the results of the investigation. To consider a subject or group as vulnerable depends on the context, and the investigator should evaluate each case individually. (REV INVEST CLIN. 2019;71:217-25)
\end{abstract}

Key words: Vulnerable groups. Women in research. Pregnant women. Low-income population. Low literacy. HIV and AIDS. Subordinates. Gender-diverse population. Informed consent. Research committee. Ethics committee. Institutional review board.

\section{INTRODUCTION}

The inclusion and protection of vulnerable populations in research and the role of Research Ethics Committees (RECs) are challenging issues. Vulnerable population groups are defined as those whose conditions hinder their access to development and improvement of their well-being. Vulnerability in research occurs when the participant is incapable of protecting his or her interests and therefore, has an increased probability of being intentionally or unintentionally harmed ${ }^{1}$; this can be due either to an inability to give informed consent or to unequal power relationships that hinder basic rights ${ }^{2}$.

It is important to note the difference between the conventional meaning of vulnerability and its special use regarding human research. Despite the vulnerability of many handicapped persons, their lack of a common capacity does not in itself point to a need for special caution on the part of the investigators. The vulnerabilities that concern us here are only those

Corresponding author:

*Alejandra González-Duarte

Departamento de Neurología y Psiquiatría

Instituto Nacional de Ciencias Médicas

y Nutrición Salvador Zubirán

Vasco de Quiroga, 15

Col. Belisario Domínguez Sección XVI, Del. Tlalpan

C.P. 14080, Mexico City, Mexico

E-mail: gonzalezduarte@aol.com

Received for publication: 21-09-2018

Approved for publication: 26-02-2019

DOI: $10.24875 / R I C .19002812$ 
Figure 1. Vulnerable groups in research. Some individuals are more vulnerable than others; this is highly dependent on the particular situation or context of the subject. Enabling tools can protect the subject and avoid his or her exclusion from a study for the sole reason of being considered vulnerable.

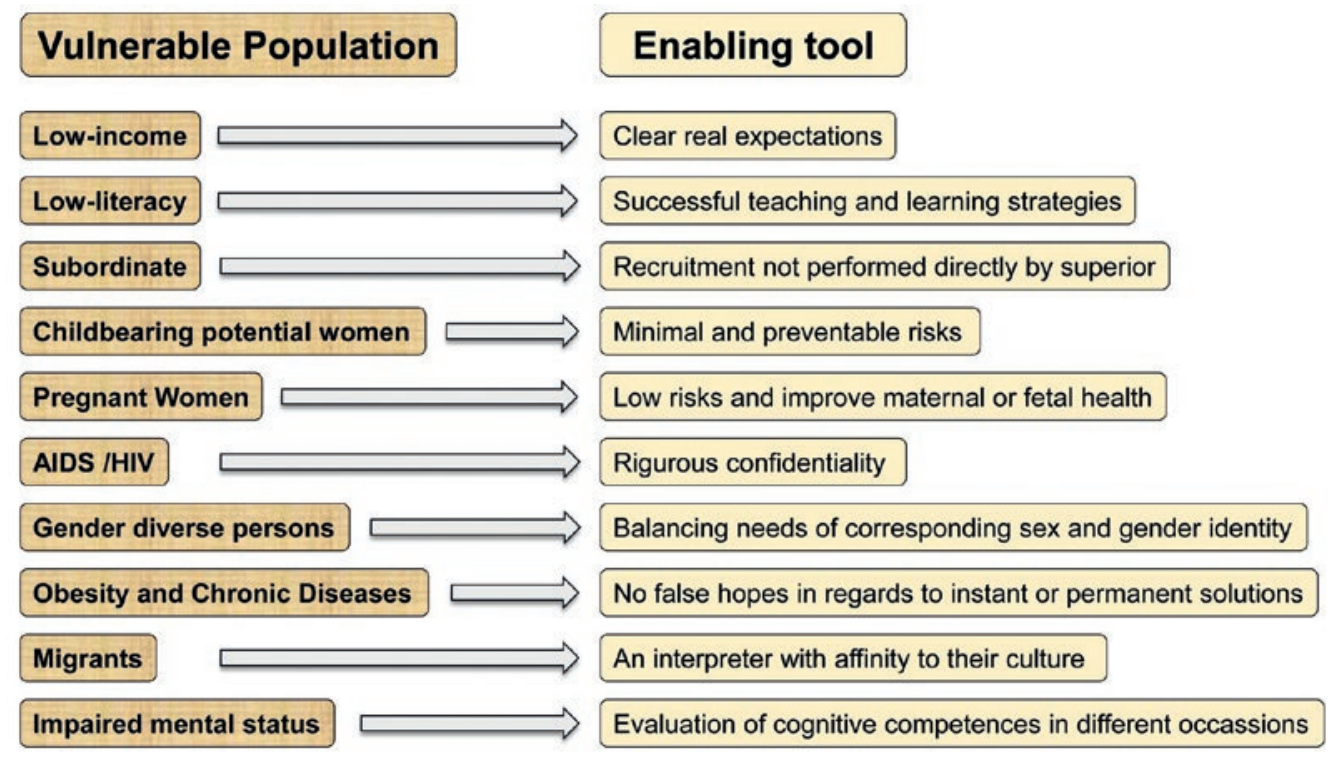

that question the efficacy of consent for the purposes of an investigation. A person who is vulnerable in the everyday sense, such as a blind person, may not necessarily be a vulnerable research subject ${ }^{3,4}$.

Socioeconomic aspects such as limited resources and lack of access to medical care can constitute vulnerability, and considering groups as vulnerable is context-dependent ${ }^{2}$. On the other hand, intrinsic factors to their situation or health condition can also cause people to be stigmatized, increasing the likelihood that others place their interests at risk, whether intentionally or unintentionally ${ }^{2}$ (Fig. 1). Although a traditional approach to vulnerability in research has been to label entire types of individuals, recently there has been a consensus that vulnerability can no longer be applied to whole groups ${ }^{2}$. The context varies between individuals and is not a static parameter in any way ${ }^{3}$. This manuscript aims to discuss the conditions that make a group vulnerable and the tools and requirements that can be used to reduce the ethical breaches when including it in research protocols. We acknowledge that not all cases of vulnerability are addressed here.

\section{LOW-INCOME POPULATIONS}

Necessity is one of the main reasons that historically have breached ethical principles in research studies.
Populations with minimal access to resources and medical treatments are vulnerable in the face of sponsors and investigators who can provide for such deficiencies. On the other hand, while it is not ethical to select communities to participate based on their unmet needs, it is also unacceptable to exclude them for the same reasons, as this will only increase the disparity. Often, when the result of the investigation is a commercial product, it will no longer be available to such communities after the study, either because it is too expensive to acquire or because the sponsor chooses a different market. It is not ethical to conduct the research, demonstrate its effects, and the potential benefits for that particular community.

\section{Tools to overcome the problem}

Ethical norms guiding research on vulnerable population groups should not be less rigorous than in any other situation ${ }^{2}$. Groups, communities, or individuals invited to participate in research should be selected based solely on scientific reasons, and not because they are easy to enroll, or because their economic or social situation makes them submissive. Some ethical principles can be observed to avoid conflict when inviting a low-income population to a scientific study ${ }^{2,5}$ : 
1. The investigation in low-income communities will not imply higher risks or burden than when it is performed in other circumstances.

2. Inclusion and exclusion criteria should not be based on discriminatory arguments such as race, ethnicity, and economic or social characteristics; if overrepresentation of a group prevails, a justification should be stated.

3. Selection of the subjects must be fair; if a subject with a higher income meets the criteria and wants to participate, he or she should not be discriminated in favor of those with a lower income.

4. Real expectations should be evident throughout the investigation, particularly when explaining the study to the subjects; no unrealistic promises must be made, for example, that completing the treatment will cure the disease or that treatment or treatment results will last forever. Clear statements should include the following considerations:

- How long will it take to finish the study and obtain the results?

- Which patients will benefit the most? (Particularly if a placebo is considered).

- What will happen after the study is finished? Will patients return to their previous conditions? Will they have access to the treatment?

- What are the risks of participating and not participating in the study?

Strategies to overcome the obstacles should be prepared beforehand, particularly regarding the plans for submission of the results to the local authorities, imposing a market price corresponding with the community standards, estimating timing to achieve availability, and contributing to social programs that may increase the likelihood of access to the studied treatment. When the product has no commercial value, but it is a relevant scientific knowledge that will change paradigms in that community, warranties must exist that results will be available as soon as possible. Additional benefits that the investigation will bring to the low-income communities, such as improvements in regional infrastructure or training of local workers, should be mentioned as surplus contributions, but clearly stating that they are not the aim of the investigation, and decisions should not be based on those benefits.

\section{LOW LITERACY POPULATION}

According to the General Conference of the United Nations Educational, Scientific, and Cultural Organization in 1978, a literate person is one who can understand a short, simple statement on his or her everyday life by reading and writing ${ }^{6}$. Many people do not achieve literacy due to inadequate schooling or even despite adequate schooling (functional illiteracy) ${ }^{7}$. Low literacy limits access to research by preventing patients from engaging fully in the process of care known as the ability to perform health-related tasks, such as taking medication, keeping appointments, preparing for tests and procedures, and giving informed consent ${ }^{8}$. Difficulties in communication arise from differences in vocabulary, grammar, and speech complexity. Subjects are less capable of asking questions or disclosing their poor understanding. Despite these difficulties, not including potential participants with a low educational level can significantly bias the results of the investigation.

\section{Tools to overcome the problem}

A limited education is not the only reason for illiteracy; other conditions such as being first-generation immigrants, elderly, or having learning disabilities account for this condition in a large proportion of cases. Fortunately, most people with low literacy skills are of average intelligence and function reasonably well by compensating for their lack of reading skills ${ }^{8}$. Given the high prevalence of low literacy in many countries around the world, mechanisms to facilitate the understanding of the research study have been designed. Some teaching and learning strategies are beneficial and therefore, are recommended in the process of inviting a patient to participate ${ }^{9}$. The teach-to-goal theory is based on a "master of learning" and postulates that all persons learn at a different speed, but anyone can master the information if given the opportunity. The relevance of this tool, particularly in low-literacy groups, has been demonstrated as a useful strategy to mitigate the differences in achieving goals matched to the level of literacy. The 
teach-back strategy consists of presenting (by the investigator) the study to the potential research participant and then asking him or her to remember and expose in his or her own words what he or she learned ${ }^{10}$; this strategy allows the investigator to understand which parts of the study were not clear enough and reeducate.

\section{SUBORDINATE SUBJECTS}

A subordinate subject is a person that has an lower rank than the investigator who will perform or promote the research ${ }^{3}$. As such, subordinates (for example, students, employees, convicts, and military) are considered as a vulnerable group; their recruitment may be the result of coercion or undue influence, or they may feel that not volunteering will negatively affect their performance, evaluations or career advancement ${ }^{11}$. For students, the pressure is generated when participation is established as a course requirement, even when alternatives are offered, since usually, these are less attractive or may require more time and effort ${ }^{12}$.

\section{Tools to overcome the problem}

It is essential that the principle of respect for persons be upheld, such that they can act autonomously ${ }^{13}$. For this, it should be first clarified that involvement in the study is entirely optional and is not a mandatory school or workplace activity, and its acceptance or rejection will not influence future practices ${ }^{13}$. Researchers must provide the potential subjects with enough information, including specific aims, anticipated benefits, possible risks, and alternative treatments, to ensure they understand all the implications of the investigation, as well as their right to withdraw at any time without penalty ${ }^{13}$.

Regarding the recruitment process, it is essential to consider two aspects: on the one hand, superiors shall not directly ask their subordinates to sign up for their research. Instead, they can post flyers or other advertisements allowing volunteers to initiate contact. Finally, confidentiality of the data collected should be ensured because the participants might know each other; likewise, the obtained results shall not be used in detriment of the individuals ${ }^{11}$.

\section{WOMEN WITH CHILDBEARING POTENTIAL}

Some researchers believe that women, in general, do not fit the definition of a vulnerable group. However, before 1993 biomedical research focused more on the health problems of men than on those of women, and women were denied access to advances in medical diagnosis and therapy as a result of being excluded from clinical trials ${ }^{14}$. Sex-dependent differences in clinical decision making and data interpretation can be crucial as they aid in the understanding of the clinical manifestations of diseases in women and their response, for example, to medications ${ }^{15}$. A significant challenge that ethics face is the risk of pregnancy for women during their participation in clinical studies.

\section{Tools to overcome the problem}

To lead to more judicious and disciplined decisions from participants, clinical studies should be given a comprehensive and preventive ethical approach to the study design, the informed consent, and the development and management of research involving this population group. Both, the researchers and the REC must consider pregnancy prevention within the structure of the project; alternatively, in the worst-case scenario, even the occurrence of an unwanted pregnancy should be taken into account. Documenting this eventuality reduces the possibility of inadequate management of the ethical conflicts that this issue entails.

The use of the selected contraceptive method and close follow-up of the adherence to it, is mandatory throughout the study. The need for legal the interruption of the pregnancy represents a relevant an severe ethical dilemma. Researchers and the local REC should take the personal points of view and judgments of the subject the study seriously. Furthermore, they must be flexible to make decisions based on the values and beliefs of the subject; for example, options for contraception should be presented $^{16}$. Compelling efforts on subject education are indicated when the proposed study presents a known threat to the reproductive system, despite the foreseeable benefit for the subject. The intensive "pregnancy prevention program" undertaken by some pharmaceutical companies serves as an example of these initiatives ${ }^{17}$. 
Figure 2. Clinical research in pregnant women. Therapeutic Research refers to procedures performed to improve maternal or fetal health. Non-therapeutic research refers to procedures that will increase scientific knowledge. Low risk is considered when the outcomes are non-existent or mild, while high risk is recognized when the outcome is moderate, severe or permanent for the mother, fetus or resulting child. Only when fetal risk is high, both maternal and paternal consent are required (if the father is present).

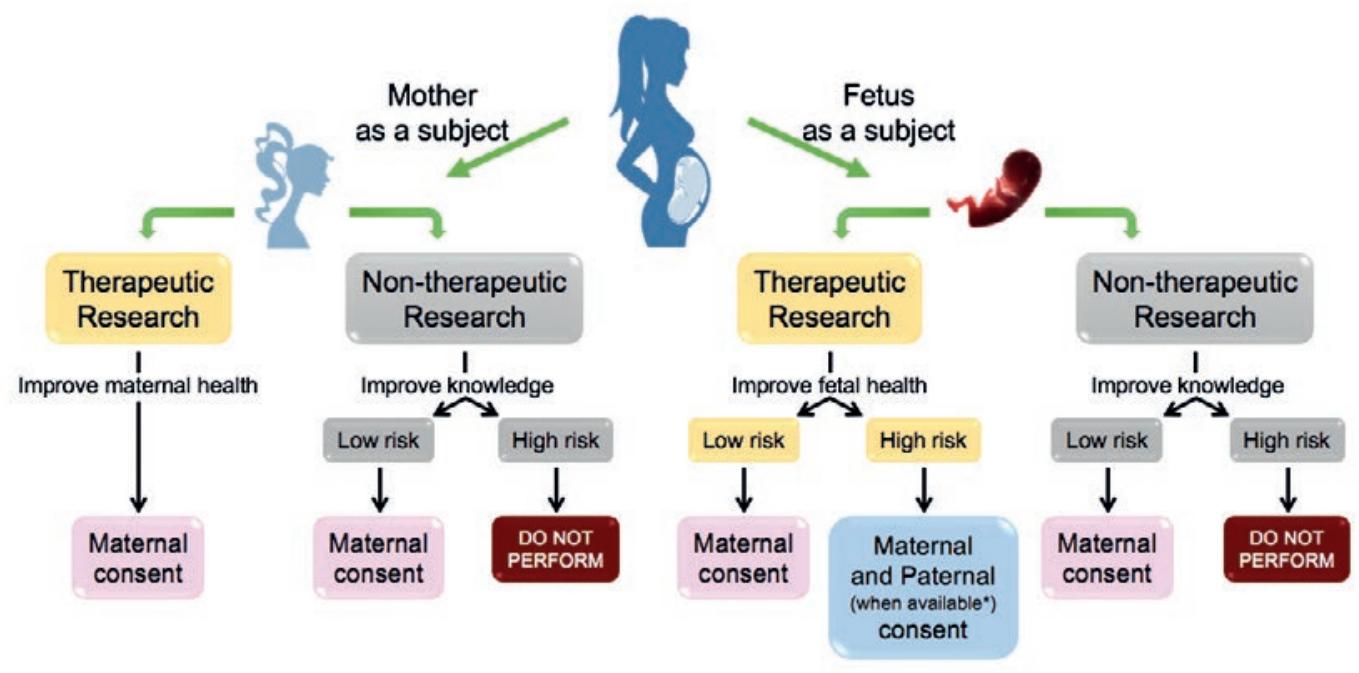

Finally, the exclusion of women with reproductive potential should be based on the argument that the risks are intolerable. The burden of argumentation in specific cases must be based on demonstrating that the balance of risks and benefits is patently unacceptable, including damage to the subject's reproductive system without compensating benefits for the subject. In all other cases, the process of informed consent must allow the woman to be the ultimate responsible for making decisions about the acceptability of risks. Last but not least, one should not forget to take a more cautious approach to research and the use of drugs in men with reproductive potential.

\section{PREGNANT WOMEN}

Participation of pregnant women in clinical trials has been a controversial issue due to potential harm to the fetus. Nonetheless, to advance prevention and treatment options for this population, pregnant women should be included in clinical research ${ }^{18}$. For ethical considerations, it is important to define who is the subject of research, the woman or the developing fetus, and make a distinction between therapeutic research (maternal and/or fetal health) and nontherapeutic research (contribution to science) ${ }^{19}$. The study design must encompass minimum risk and maximum benefit (Fig. 2).

- The mother as a subject: ethical considerations regarding a woman's participation in studies that involve treatment will depend not only on whether the treatment will affect her health but also on the type and magnitude of the benefit, the risk to her offspring, and the balance between the maternal health needs and the offspring risk ${ }^{19}$. For example, using established or experimental therapies for treating morning sickness during pregnancy is a health need, but if the treatment is teratogenic, it is unethical to provide it, even if it improves women's health ${ }^{19}$.

- Therapeutic research on the mother: pregnant women may participate in clinical research when the purpose of the study is to improve maternal health, despite a fetal risk.

- Non-therapeutic research on the mother: if the clinical research does not have the objective to meet the mother's health needs, the study should only be performed if the risk to the fetus, and the resulting child, is minimal. It would be unethical to forfeit the interests and health of the woman's offspring by contributing to science advancement. 
- The fetus as a subject: research performed on developing embryos or fetuses should also focus on the child (if carried to term), and the effects that prenatal interventions will have on offspring.

- Therapeutic research on the fetus: experimental procedures designed to prevent or treat disability or disease in offspring should be permitted.

- Non-therapeutic research on the fetus: non-therapeutic research on the fetus is permitted when the risk to the fetus is minimal, and the purpose of the activity is to contribute to science and biomedical knowledge that cannot be obtained by other means ${ }^{19}$.

\section{Tools to overcome the problem}

To make research ethically acceptable on fetuses that will be aborted, the experimental procedure should be administered immediately before the abortion is performed or in other circumstances in which it is evident that the pregnancy will be terminated. The investigator should be assured that the woman had sufficient time and opportunity to consider her decision. Researchers should not be allowed to be involved in decisions regarding termination of pregnancy or viability of the fetus ${ }^{19}$.

Another ethical concern is who must give informed consent (Fig. 2). The requirement of paternal consent remains a barrier to participation in clinical research in pregnant women. An exception occurs when the potential benefit for the fetus is clearly higher than the risk; then, only maternal consent is required ${ }^{20}$.

\section{PERSONS LIVING WITH HIV OR AIDS}

The case of HIV/AIDS includes individuals and population groups with a wide range of vulnerability characteristics, such as belonging to racial/ethnic minorities, being women, having different sexual preferences, being sexual workers, and/or using drugs ${ }^{21}$. However, vulnerability as a group arises from the risk of social stigmatization and issues related to employment and insurance that the subjects may face if their status is revealed.

\section{Tools to overcome the problem}

The ethical and research committees and the community advisory committees should oversee that proper handling of those conflicts is considered since the design and planning stages and throughout the conduction and analysis of the study. Confidentiality should be rigorous and should include notification of seropositivity, counseling, and safeguarding the information of sensitive topics regarding the subject's behaviors in any of the following issues: sexual orientation, incest, rape, sexual molestation, deviant sexual behavior or attitudes regarding misconduct, pedophilia, bestiality, etc.; practices of contraception, abortion and/or pregnancy; substance use and/or abuse including prescription medications legally or illegally obtained; mental health, for example, suicide attempt, depression, and gambling; illegal or taboo behavior; and traumatic experiences. The investigator should not express his or her opinion on any of these subjects verbally or non-verbally and should refrain from making a comment or repeating the information obtained ${ }^{22}$.

\section{GENDER DIVERSITY}

Gender-diverse persons include different types of individuals. There is no precise definition for this population group, and there is a lack of specificity of these terms. Data show that between $1 \%$ and $10 \%$ of individuals consider themselves to belong in this group, although the true proportion of the population classified in the different sexual orientations is unknown. This wide range of possibilities reflects the difficulties in obtaining information, therefore, indicating the lack of data on how gender-diverse persons have been represented in research studies. Basic needs such as regular screening tests may not be performed due to a mismatch between the subject's gender identification and the screening protocol, for example, prostate screening in men identified as women or cervical cancer smears in women identified as men ${ }^{23}$.

\section{Tools to overcome the problem}

From a medical perspective, research is necessary to explore which gender will be used for demographics in the study protocol or how to introduce sex-oriented recommendations without incurring in lack of 
sensibility, for example, proposing the use of oral contraceptives in women identified as men ${ }^{24}$.

\section{OBESITY AND CHRONIC DISEASES}

Participants with obesity and chronic diseases are frequently discriminated or stigmatized due to their physical appearance. Moreover, they often receive false hope, or have been misled or deceived by health professionals. They have been offered costly treatments which are ultimately ineffective and even dangerous, thus taking advantage of the subject's need for rapid solutions to a complicated and frustrating health situation, and magnifying their vulnerability. The case of patients with obesity is paramount.

Studies undertaken in schools or other settings involving children or adolescents with obesity pose a number of ethical considerations beyond their already vulnerable status of belonging to these age groups. Several questions should be considered: was the whole class or school selected? Were the individuals selected based on their weight status? Will the intervention be carried out in a public space? Is privacy guaranteed? Children or adolescents with obesity may suffer from stigmatization and bullying if their rights as study subjects are not adequately protected. Ethical issues also arise when inviting subjects to participate in research studies. Dilemmas emerge in clinical trials where novel treatments, including pharmacological agents - usually expensive -, to promote weight loss are studied. When considering the need for prolonged treatment due to the nature of the disease, if the therapy proves beneficial will the participant have access to it once the trial is over? Will it be readily available for participants in the control group?

\section{Tools to overcome the problem}

The problem of overweight and obesity due to its complexity requires multidimensional solutions. It may be challenging to justify intervening in the lives of some individuals or groups in the interest of achieving better health outcomes for the whole population $^{21}$, since unintended effects of research studies and programs, such as stigmatization and discrimination, are not uncommon ${ }^{23}$.
Ethical considerations arise regarding access to preventive treatments and the justification for intervening in the private lives of individuals with overweight or obesity, mainly when financial resources are limited, and evidence about the long-term impact of obesity interventions is uncertain. Intervention in individuals with excessive weight has been justified with the argument that doing so will have a positive effect not only on individuals but also on their families and public health in general while, at the same time, will improve socal costs and have a positive impact on the healthcare system. This is a sensitive matter since health promotion strategies that emphasize the responsibility to make healthy or accepted choices (being fitter, thinner, stronger, faster, and smarter), can lead to punitive consequences for those who make unhealthy, over-consumptive choices (of food, alcohol, etc.,) and can hinder the individual's autonomy ${ }^{26,27}$.

\section{MIGRANTS}

Migrant populations face multiple challenges to have access to health services, leaving them at risk of being subject of unethical conducts (i.e., exploitation, exclusion, discrimination, and violence $)^{28}$. Although migrant groups are heterogeneous, they have several vulnerability traits in common, such as acute malnutrition, transmissible infectious diseases or non-communicable diseases, or being victims of organ trafficking. Despite the currently growing number of migrants, bioethical debates have rarely focused on this topic. Critical issues remain unsolved such as the responsibility of a country to provide health services to their illegal immigrants. Moreover, this population is usually afraid of participating in research studies since their status as citizens could be compromised; therefore, broadening the disparities between citizens and immigrants ${ }^{29}$.

\section{Tools to overcome the problem}

Health services should adjust their procedures to ensure that all migrants preserve respect to their autonomy and that all have access to an interpreter when invited to participate in a research study. Laws should be updated regularly to ensure the protection of the migrants on the current circumstances. No mandatory testing for diseases is acceptable, unless there are epidemiological reasons to protect 
them or other members within the community. For research studies, migrants should be a matter of protection programs (especially children, orphans, the elderly, and handicapped). Researchers should identify the challenges that this group may face to provide informed consent. False promises or manipulations of the benefits and risks are common deviations that should be avoided. In some instances, RECs may consider including a member of the migrant community to represent the culture of their group fully ${ }^{30}$.

\section{PERSONS WITH IMPAIRED MENTAL STATUS}

With increasing knowledge on the field and newly developed technology, dementias and other syndromes of cognitive decline are being diagnosed earlier throughout the disease. Thus, cognitive impairment may be mild, moderate or severe, and slow, stable or rapidly progressive, denoting that dementia per se does not imply a loss of all cognitive functions, nor loss of the capacity to make all decisions ${ }^{31}$. The ability to understand and consent to participate in a research trial is directly proportional to the complexity of the decision and the understanding that the person has of its consequences ${ }^{32,33}$. There is no specific threshold to establish whether a patient can or cannot consent, and all cases should be evaluated individually. In addition to diminished mental capacity, other characteristics must be considered when declaring a patient unfit for clinical investigations, such as fluctuations in mental status from day to day, inability to communicate verbally or non-verbally, and decreased awareness of suffering from a disease ${ }^{34}$.

\section{Tools to overcome the problem}

To respect the principle of autonomy, the diagnosis of functional capacity should not be based solely on the diagnosis of dementia, but specific tests to evaluate the different competencies should be used ${ }^{35}$ :

- Informed capacity: can the subject understand what is being discussed, for example, the nature of the procedure (surgery vs. a medical procedure)?

- Cognitive and affective capacity: can the patient understand the different alternatives that may exist? Can the patient point towards the most probable outcome according to each option? For example, if he or she does not undergo surgery, will something happen?

- Choosing capacity: can the person decide between different options and indicate which one he or she prefers? Can the person justify his or her election? Is this decision sustained over time?

- Understanding the decision capacity: can the person explain the process to choose?

Some additional tools can evaluate the ability of a person to make rational decisions, such as Whites Criteria or MacArthur Competence Assessment Tool for Clinical Research ${ }^{31}$.

\section{CONCLUSIONS}

The vulnerability is a central concept in protecting human subjects in research, and the term, vulnerable populations, was introduced as part of the guidelines for medical ethics in the 1949 Nuremberg Code, updated in the 2008 World Medical Association Declaration of Helsinki, and in the 1979 Belmont Report to protect human subjects involved in research $^{36}$. Excluding subjects from research for the sole reason of belonging to a vulnerable group are unethical and will bias the investigation's results. However, it is indisputable that the investigator and the RECs must consider additional protection for vulnerable populations. They must ensure a full understanding by the subjects of the information given when inviting them to participate in a study protocol; ensure that the study subject is not selected or rejected for the wrong reasons and that there are no secondary or hidden interests when performing the research. Specific tools can be used for this purpose. The limitations of this manuscript include the different legislation and regulations that exist as well as the patients' individual beliefs that should be considered for each case.

\section{ACKNOWLEDGMENTS}

Ethics Committee members: Arturo Galindo Fraga (Department of Hospital Epidemiology and Quality Control 
of Medical Care), Sergio Hernández Jiménez (Center for the Comprehensive Care of the Patient with Diabetes), Alejandra González-Duarte (Neurology and Psychiatry Department), Elena Zambrano González (Department of Reproductive Biology), Mauricio Sierra Salazar (Surgery Division), Patricia Domínguez Sánchez (Nursing Educational Department), Jorge Oseguera Moguel (Cardiology Department), Álvaro Aguayo González (Hematology and Oncology Department), and Alejandra Armenta Espinoza (External Member), Instituto Nacional de Ciencias Médicas y Nutrición Salvador Zubirán, Mexico City, Mexico.

Research Committee members: Carlos A. Aguilar Salinas (Metabolic Diseases Research Unit and Endocrinology Department), Marta Durand Carbajal (Department of Reproductive Biology), Martha KauferHorowitz (Obesity and Eating Disorders Clinic), Josefina Alberú Gómez (Department of Transplantation), Alvar Loria Acereto (Clinical Epidemiology Unit), Marina Rull Gabayet (Rheumatology Department), Heriberto Medina Franco and Carlos Hinojosa Becerril (Surgery Division), Instituto Nacional de Ciencias Médicas y Nutrición Salvador Zubirán, Mexico City, Mexico.

\section{REFERENCES}

1. Levine C, Faden R, Grady C, et al. The limitations of "vulnerability" as a protection for human research participants. Am J Bioeth. 2004;4:44-9.

2. Council for International Organizations of Medical Sciences $(\mathrm{Cl}-$ OMS). International Ethical Guidelines for Health-Related Research Involving Humans. $4^{\text {th }}$ ed. Geneva: Council for International Organizations of Medical Sciences (CIOMS); 2016.

3. Biros M. Capacity, vulnerability, and informed consent for research. J Law Med Ethics. 2018;46:72-8.

4. Blustein J. The history and moral foundations of human-subject research. Am J Phys Med Rehabil. 2007;86:82-5.

5. Tamariz L, Palacio A, Robert M, Marcus EN. Improving the informed consent process for research subjects with low literacy: a systematic review. J Gen Intern Med. 2013;28:121-6.

6. Vágvölgyi R, Coldea A, Dresler T, Schrader J, Nuerk HC. A review about functional illiteracy: definition, cognitive, linguistic, and numerical aspects. Front Psychol. 2016;7:1617.

7. Wendler D. Can we ensure that all research subjects give valid consent? Arch Intern Med. 2004;164:2201-4.

8. Roter DL, Rudd RE, Comings J. Patient literacy. A barrier to quality of care. J Gen Intern Med. 1998;13:850-1.

9. Flory J, Emanuel E. Interventions to improve research participants' understanding in informed consent for research: a systematic review. JAMA. 2004;292:1593-601.

10. Kripalani S, Bengtzen R, Henderson LE, Jacobson TA. Clinical research in low-literacy populations: using teach-back to assess comprehension of informed consent and privacy information. IRB. 2008;30:13-9.

11. Schwenzer KJ. Protecting vulnerable subjects in clinical research: children, pregnant women, prisoners, and employees. Respir Care. 2008;53:1342-9

12. Leentjens AF, Levenson JL. Ethical issues concerning the recruitment of university students as research subjects. J Psychosom Res. 2013;75:394-8.

13. Spence DL. Ensuring respect for persons when recruiting junior enlisted personnel for research. Mil Med. 2007;172:250-3.
14. Wizemann TM, Pardue ML. Exploring the Biological Contributions to Human Health Does Sex Matter? Institute of Medicen (US) Committee on Understanding the Biology of Sex and Gender Differences. Wasington, DC: National Academies Press; 2001.

15. Liu KA, Mager NA. Women's involvement in clinical trials: historical perspective and future implications. Pharm Pract (Granada). 2016;14:708.

16. McCullough LB, Coverdale JH, Chervenak FA. Preventive ethics for including women of childbearing potential in clinical trials. Am J Obstet Gynecol. 2006;194:1221-7.

17. Mastroianni AC, Faden R, Federman D. Women and Health Research: ethical and Legal Issues of Including Women in Clinical Studies. Institute of Medicine (US) Committee on the Ethical and Legal Issues Relating to the Inclusion of Women in Clinical Studies. Washington DC: National Academies Press (US); 1999.

18. Frew PM, Saint-Victor DS, Isaacs MB, et al. Recruitment and retention of pregnant women into clinical research trials: an overview of challenges, facilitators, and best practices. Clin Infect Dis. 2014;59 Suppl 7:S400-7.

19. Mastroianni AC, Faden R, Federman D. Workshop and commissioned papers. Institute of medicine (US) committee on the ethical and legal issues relating to the inclusion of women in clinical studies. In: women and Health Research: ethical and Legal Issues of Including Women in Clinical Studies. Vol. 2. Washington, DC: National Academies Press (US); 1999.

20. Rachin JD. A Report of Task Force Sponsored by the NIH Office of Research on Women's Health. Science Meets Reality: recruitment and Retentions of Women in Clinical Studies, and the Critical Role of Relevance. Ch. 4. Informed Consent; 2003.

21. Sengupta S, Lo B, Strauss RP, Eron J, Gifford AL. How researchers define vulnerable populations in HIV/AIDS clinical trials. AIDS Behav. 2010;14:1313-9.

22. Marlboro College. Vulnerable Populations and Other Ethical Considerations. Available from: https://www.nook.marlboro.edu/ public/academics/irb/vulnerable populations\#hiv.

23. Marshall Z, Welch V, Thomas J, et al. Documenting research with transgender and gender diverse people: protocol for an evidence map and thematic analysis. Syst Rev. 2017;6:35.

24. Bowman SM. Lesbian, Gay, Bisexual, Transgendered, and Queer Populations: challenges in Recruitment, Retention, and Relevance. Science meets reality: recruitment and Retention of Women in Clinical Studies, and the Critical Role of Relevance. Vol. 8. A Report of the Task Force Sponsored by the NIH Office of Research on Women's Health NIH Publication No. 03-5403; 2003. p. $115-7$

25. Cochrane Library. Prevention of Obesity. Special Collection. Available from: http://www.thecochranelibrary.com/details/collection/1417657/Prevention-of-obesity.html. [Last accessed on 2011 Dec 07].

26. Fry CL. Competing interests, intervention limits, and permissible health identities. Health Promot J Aust. 2010;21:170-5.

27. Hurlimann T, Peña-Rosas JP, Saxena A, Zamora G, Godard B. Ethical issues in the development and implementation of nutrition-related public health policies and interventions: a scoping review. PLoS One. 2017;12:e0186897.

28. Klingler C, Odukoya D, Kuehlmeyer K. Migration, health, and ethics. Bioethics. 2018;32:330-3.

29. Eckenwiler L, Straehle C, Chung R. Global solidarity, migration and global health inequity. Bioethics. 2012;26:382-90.

30. World Health Organization. Health of Refugees and Migrants. Available from: http://www.who.int/migrants/publications/ EMRO-Practices.pdf. [Last accessed on 2019 Feb 06].

31. Arenas Massa A, Slachevsky Chonchol A. Decision making and consents given by demented patients. Are they valid? Rev Med Chil. 2017;145:1312-8.

32. Radoilska L. Introduction: personal autonomy, decisional capacity, and mental disorder. In: autonomy and Mental Disorder Oxford, UK: Oxford University Press; 2012. p. 9-41.

33. Warner J, McCarney R, Griffin M, Hill K, Fisher P. Participation in dementia research: rates and correlates of capacity to give informed consent. J Med Ethics. 2008;34:167-70.

34. Trachsel M, Hermann H, Biller-Andorno N. Cognitive fluctuations as a challenge for the assessment of decision-making capacity in patients with dementia. Am J Alzheimers Dis Other Demen. 2015;30:360-3.

35. Alvaro LC. Competency: general principles and applicability in dementia. Neurologia. 2012:27:290-300

36. The Belmont Report: three Principles for Ethical Research. Available from: https://www.msalganik.wordpress.com/2014/07/ 29/the-belmont-report-three-principles-for-ethical-research. [Last accessed on 2019 Feb 06]. 